\title{
COMMISSION 7: CELESTIAL MECHANICS AND DYNAMICAL ASTRONOMY
}

\author{
(MECANIQUE CELESTE ET ASTRONOMIE DYNAMIQUE)
}

\author{
PRESIDENT: Claude Froeschlé \\ VICE-PRESIDENT: John Hadjidemetriou \\ ORGANIZING COMMITTEE: R. Dvorak, S. Ferraz-Mello, T. Fukushima, \\ I.A. Gerasimov, D.C. Heggie, Z. Knezevic, J. H. Lieske, A. Milani, J.C. Muzzio, \\ M. Soffel \& Y. Sun \\ SECRETARY: A. Lemaitre
}

\section{INTRODUCTION}

Research in Celestial Mechanics, for the past three years, has mainly focused on the understanding of Chaos on all its aspects. The always larger number of potential applications (meteors, KBO, NEA, asteroids of the main belt but also exoplanets or galactic motions) and the development of new efficient tools, like the symplectic integrators, have allowed the passage from QUALITATIVE models (for example the transfer mechanisms) to real QUANTITATIVE results (like the calculation of lifetimes) . This important step has contributed to (re)create collaborations between theoreticians and observers (for example, in the prediction of catastrophic impacts) and to situate the Celestial Mechanics in a wider scientific context.

\section{SCIENTIFIC HIGHLIGHTS 1997-1999}

\subsection{Dynamics of the Asteroid Belt}

\section{Author of the report : N. MURRAY}

The asteroid belt has supplied us, in the form of meteorites, with our only samples of primordial solar system material, and hence revealed to us the age of the Earth and Sun. It also serves as a reservoir for crystals formed in the envelopes of evolved stars, which again are delivered to Earth in meteorites. Objects from the belt may be implicated in past extinction events on Earth. The belt is the probable source of near-Earth asteroids, some of which might cause extinction events in the future. Transport of material from the asteroid belt to the Earth is clearly important, but only in the last few years have we begun to understand in detail how such transport arises.

The first hints came from the work of Wetherall and Williams (1979, Origin and distribution of the elements, L.H. Ahrens ed. Pergamon press, Oxford) and Wisdom (1985, Nature 315, 731). Wetherall and Williams showed that a resonance between the precession rate of an asteroid's orbit and that of the secular frequency $g_{6}$ (appearing in the precession of Jupiter and Saturn) would lead to a rapid growth in the eccentricity of the asteroid's orbit. In a short time the asteroid orbit would cross that of Mars and even Earth, suggesting one possible route from the belt to the inner solar system. Wisdom found that bodies with orbital periods equal to one third that of Jupiter (a $3 / 1$ resonances) were also unstable to an increase in orbital eccentricity, suggesting a second route. For a time, it appeared that some combination of these paths would explain the origin of meteorites, although the number of large bodies in near-Earth orbits was harder to understand.

However problems with both scenarios soon became apparent. Measurements of the cosmic ray exposure times of ordinary meteorites (chondrites), which rely on the generation 
of radioactive elements in the meteor by high energy cosmic rays, showed that most chondrites took of order 20 million years to reach the Earth. Brute force numerical integrations, made possible by a combination of improved computers and better integration algorithms (symplectic integrators) then showed that the life time of objects in either the $3 / 1$ resonance or the $\nu_{6}$ resonance (involving $g_{6}$ ) is one million years or less (Gladman 1996, Ph.D. thesis, Cornell University). Most objects in either resonance fall into the Sun. Another problem was that the flux of bodies injected into either type of resonance, presumably by collisions, was apparently too low to maintain either the meteorite or near-Earth asteroid population. Both difficulties suggested that there was more to the story, in particular, that there were other roads from the belt to the inner solar system.

The $3 / 1$ resonance clearly operated to remove bodies. Kirkwood (1867) pointed out a lack of asteroids with that orbital period more than 100 years ago. He also noted the presence of similar gaps at other low order resonances. Numerical work by numerous groups (Milani, C.D. Murray, Nobili, Wisdom) indicated that the gaps were caused by increases in eccentricity due to chaotic motion forced by the perturbations of Jupiter. The motion was known to be chaotic because the integrations showed that asteroids in the resonances had positive Lyapunov exponents; nearby orbits diverged from each other exponentially with time. However, a quantitative theoretical understanding of the origin of the chaos was lacking. Neither the Lyapunov exponent, nor the lifetime of the asteroid, could be calculated analytically.

Work by Morbidelli et al. (1995, Icarus 118, 132) provides observational evidence that high order resonances, in particular the $9 / 4$ (5th order) resonance could affect the orbits of asteroids in the belt. Holman and Murray $(1996, A J 112,1278)$ suggested that actual gaps, similar to the classical Kirkwood gaps, existed at the $9 / 5,7 / 4,5 / 3$, and $8 / 5$ resonances, with a possible gap at the location of the 11/6 mean motion resonance with Jupiter. Holman and Murray also gave an explicit analytic calculation of the Lyapunov exponent for a general mean motion resonance. The theory explained the origin of the chaos in mean motion resonances in terms of the overlap of the components of the resonances.

Murray and Holman $(1997, A J 114,1246)$ presented an analytic theory of the life time of asteroids placed in two body mean motion resonances in the three body problem. Overlap of the components of a mean motion resonance insures that the resonant angle acts like a random variable. This random variable acts as a source of noise in the dynamics describing the orbital angular momentum, forcing the eccentricity of the asteriod to undergo a random walk. The step size is proportional to the square root of the strength of the resonance $\left(\sqrt{M_{J} e^{q}}\right.$ for a $q$ th order resonance with Jupiter), and a time between steps of order the Lyapunov time. An asteroid placed in a mean motion resonance in the outer belt is removed when its eccentricity random walks up to the value at which the orbit crosses that of Jupiter; it is then quickly scattered out of the solar system by close encounters with that planet. Asteroids in the inner belt random walk until they encounter either the $\nu_{6}$ resonance, or the orbit of Mars or Earth; they are fated to strike the sun, or less commonly, one of the terrestrial planets.

This theory worked well for mean motion resonances with $q>1$, but more subtle efforts are probably required for first order resonances. The theory does not account for the bulk of the chaos seen in integrations accounting for the effects of all the giant planets, as opposed to those including only Jupiter. Holman and Murray showed numerically that unlike the three body model, models involving more than one giant planet cause chaos almost everywhere in the outer belt.

The later shortcoming of the theory, the inability to explain chaos not associated with two body mean motion resonances, was quickly remedied. Murray et al. (1998, $A J$ 116, 2583) and Nesvorny and Morbidelli (1998, $A J$ 116, 3029 and 1998, CM 71, 243) showed that three body mean motion resonances, involving two planets and an asteroid, cause the bulk of the chaos seen in integrations of the asteroid belt. Numerical work by Ferraz-Mello et al. $(1998, A J 116,1491)$ shows that a three body resonance is likely to play a role in depleting the $2 / 1$ two body resonance! 
Murray et al. (1998, $A J 116,2583)$ show that, as in two body resonances, the asteroid's eccentricity undergoes a random walk. In the outer belt the life times generally exceed the age of the solar system. In the inner belt this is not the case. This is emphatically not the case in the inner belt; as speculated by Miglorini et al./ (1998, Science 281, 2022), numerical work by Morbidelli and Nesvorny $(1998, A J 116,3029)$ strongly suggests that near-Earth asteroids are bodies that previously resided in the inner asteroid belt. The original orbits were unstable due to three body resonances between the asteroid, Jupiter, and Mars. The asteroid's orbital eccentricity increased until the orbit crossed that of Mars. Subsequently the asteroid semimajor axis undergoes a random walk until the object becomes a near-Earth asteroid. Morbidelli and Nesvorny speculate that the Late Heavy Bombardment of the early Solar System (suggested by the large number of very old craters seen on the Moon) might be due to the early depletion of a population of moderately high eccentricity asteroids via this mechanism.

The situation today is in some respects the reverse of that after the work of Wisdom and Wetherall and Williams fifteen to twenty years ago. We have a promising but relatively untested theory (three-body resonances) for the origin of near-Earth asteroids, while the delivery mechanism for meteorites is harder to understand. Two body resonances move material on too short a time, while three body resonances take too long. It would appear that non-gravitational forces might need to be invoked to explain the 20 million year delivery times indicated by cosmic ray exposure times.

\subsection{Dynamical Transfer in the Solar System}

\section{Author of the report: B. GLADMAN}

This report covers advances in the study of natural dynamical transport of small bodies in the solar system (thus neglecting planet migration studies), for material published in refereed journals between 1996 and mid-99. The bulk of the advances in this area were, like in the 3-year period preceding it, driven by the powerful combination of cheap, fast computer hardware and the availability of the efficient algorithms based on the so-called 'mixed variable symplectic' integration algorithms. The first of these was presented by Wisdom and Holman in $(1991, A J \mathbf{1 0 2}, 1528)$, and gave about a 30-100 times computational speed improvement for qualitative explorations of dynamical orbital stability in heliocentric problems (those in which there is a central gravitationally dominant object). In 1994 Levison and Duncan presented a modified form of this algorithm that was able to cope with close approaches between test particles and the planets (a case where the original algorithm did not function well); it would be fair to say that this algorithm has revolutionized the topic by allowing the direct simulation of the orbital evolution of statistically meaningful numbers of particles over timescales comparable to their dynamical lifetimes, and that the majority of the advances in this field (the study of transport from one region of the Solar System to another) in the last 3 years have come about because of these algorithms. Note that this review is primarily concerned with transport studies rather than stability or mapping of resonant phenomena.

Levison and Duncan (1997, Icarus 27, 13) modelled the transport of trans-neptunian objects from the Kuiper belt down through the Centaur region to the short-period comet domain. Duncan and Levison $(1997$, Science 276, 1670) also used the results of these experiments to discover that a reasonable fraction (of order 1 percent) were 'scattered' out to large semimajor axes (hundreds of $\mathrm{AU}$ ) on orbits surviving the age of the solar system. Morbidelli $(1997$, Icarus 127, 1) examined the transport of comets out of the 2:3 resonance in the Kuiper Belt to planet-crossing orbits as an additional supply mechanism. Dones (1996, PASP CS 07, 233) studied the transport and dynamical lifetimes of Centaurs in the outer solar system.

The transport of asteroidal material out of the asteroid belt was the subject of many papers during this period. Moons $(1997, C M 65,175)$ reviewed the literature regarding resonant pumping of objects to planet-crossing orbits. The so-called GAPTEC project (Gladman et al. 1997, Science 277, 197) directly integrated particles emerging from all 
of the main asteroid-belt resonances and established their dynamical lifetimes; this study examined the transport of such material into near-Earth orbits, concluding that because the phenomena of Sun-grazing (orbital eccentricity driven to unity) it is very important that a larger injection rate is required to maintain the near-Earth asteroid (NEA) population in a steady state. The importance and efficiency of the Mars-crossing population in supplying NEAs was the subject of Migliorini et al. (1998, Science 281, 2022), which showed that the transport of very large $(>5 \mathrm{~km})$ NEAs to Earth-crossing is likely dominated by asteroids that spend long periods in Mars-crossing orbits before reaching Earth-crossing space, rather than coming 'directly' from the main resonances. Morbidelli and Nezvory (1999, Icarus 139, 295) showed that this Mars-crossing population could in turn be kept in steady state by 'three-body' resonances in the inner asteroid belt. The possibility that some of the NEA population consists of cometary bodies transported to orbit entirely interior to Jupiter's orbit was studied by Harris and Bailey (1996, Irish $A J$ 23, 151 and 1998, MNRAS 297, 1227 ), who showed that purely gravitational mechanisms seem to be insufficient to explain the existence of near-Earth objects like comet Encke, but that non-gravitational mechanisms might plausibly work; further work is needed.

An associated group of papers concerned the transport of asteroidal material directly to Earth (rather than near-Earth space) in the context of meteoritical studies. Migliorini et al. (1997, MPS 32, 903) studied the transport of HED meteorites from Vesta. Morbidelli and Gladman $(1998, M P S 33,999)$ matched the observed orbital distribution of incoming chondritic fireballs with a dynamical model of an asteroidal source; although matching the orbital distribution well, this study predicted a time scale problem for meteoritical delivery (with meteorites arriving at the Earth 3 to 10 times faster than their cosmic-ray exposure ages indicate). A solution to this paradox has been suggested by the Yarkovsky drift, which could transport meteoroid-sized bodies to the resonances on the correct time scales; see Vokrouhlicky and Farinella (1998, $A J$ 116, 2032), Farinella et al. (1998, Icarus 132, 378 ), and references to previous papers therein. The concept of very large impactors being transported to the Earth in 'asteroid showers' was the subject of Zappala et al. (1998, Icarus 134, 176).

The transport of planetary ejecta between planets (with particular attention to the lunar and martian meteorites) was the subject of two papers (Gladman et al. 1996, Science 271, 1387 and Gladman 1998, Icarus 130, 228); transport efficiencies were quantified and timescales were shown to agree with cosmic-ray exposure studies of these meteorites.

Work has continued on the transport of dust in the solar system, both from the asteroid belt (Kortenkamp and Dermott 1998, Icarus 135, 469) and now from the Kuiper Belt (Liou and Zook 1999, $A J$ 118, 580 and Gorkavyi et al. 1997, ApJ 488, 268) and references in those papers. Many papers covering the production rate (but not transport) of dust will not be discussed here.

\subsection{Symplectic Integrators}

Author of the report: J. E. CHAMBERS

Symplectic integrators precisely follow the evolution of a Hamiltonian system designed to be very similar to the problem of interest. As a result, these integrators show no long-term build up in energy or angular momentum errors except due to computer roundoff. Symplectic algorithms are most efficient for Hamiltonians $H=H_{0}+H_{1}$ where $\epsilon=H_{1} / H_{0} \ll 1$, in which the system can be advanced efficiently under each part of $H$ separately. In celestial mechanics, $H_{0}(\mathbf{p}, \mathbf{r})$ can be the Keplerian motion of each object about the central body, and $H_{1}(\mathbf{r})$ the perturbations between bodies (Wisdom and Holman 1991, $A J 102,1528$ ), where $r$ and $p$ are the coordinates and momenta.

The most widely used algorithm is the second-order 'leapfrog' method. One leapfrog timestep is

$$
S_{2}=H_{1}[\tau / 2] H_{0}[\tau] H_{1}[\tau / 2]=H[\tau]+O\left(\epsilon \tau^{3}\right)
$$

where $H_{A}\left[\tau_{B}\right]$ implies advancing the system under Hamiltonian $H_{A}$ for a time $\tau_{B}$. 
Higher-order methods are given in Yoshida (1990, Phys. Lett. A. 150, 262). Recently, Chambers and Murison (1999, $A J$ in press) described a more efficient class of 'pseudo-order' methods, designed for cases when $\epsilon \ll 1$. A pseudo-4th-order method is

$$
S_{4 *}=H_{1}[\tau / 6] H_{0}[\tau / 2] H_{1}[2 \tau / 3] H_{0}[\tau / 2] H_{1}[\tau / 6]=H[\tau]+O\left(\epsilon \tau^{5}\right)+O\left(\epsilon^{2} \tau^{3}\right)
$$

The second-order method can be improved substantially using 'symplectic correctors' (Wisdom et al.; 1996, Fields IC 10, 217). These initially convert from physical to integration' variables. Prior to output or the end of the integration the reverse correction is applied. Each step using correctors is equivalent to

$$
S_{2, \text { cor }}=C^{-1} S_{2} C=H[\tau]+O\left(\epsilon^{2} \tau^{3}\right)
$$

where $C=H_{0}\left[\tau_{1}\right] H_{1}\left[\tau_{2}\right] \ldots H_{0}\left[\tau_{n-1}\right] H_{1}\left[\tau_{n}\right]$.

The symplectic algorithms can be modified to include dissipative forces (Malhotra 1994, CM 60, 373, Cordeiro et al./, 1997, CM 65, 407), or relativistic corrections (Saha and Tremaine 1994, $A J$ 108, 1962; these authors also describe how to give individual timesteps to different bodies). Mikkola $(1998, C M 68,249)$ has described a general method for including any velocity-dependent forces into analogues of the symplectic integrators.

The fixed stepsize of symplectic integrators makes them inefficient for eccentric orbits. Rauch and Holman $(1999, A J 117,1087)$ show that the leapfrog method becomes unstable in highly eccentric cases unless $\tau$ is very small. If the timestep is varied adaptively, the integrator no longer behaves symplectically (Gladman et al., 1991, CM 52, 221 and Michel and Valsecchi 1997, CM 65, 355). Mikkola $(1997, C M 67,145)$ has shown how to overcome this problem using time regularization $d t=g(r) d s$ and extended phase space $\left(\mathbf{r}, \mathbf{p}, r_{0}=\right.$ $\left.t, p_{0}=H\right)$, The new Hamiltonian is $\Gamma=\Gamma_{0}\left(\mathbf{p}, \mathbf{r}, p_{0}\right)+\Gamma_{1}(\mathbf{r})$, where $\epsilon=\Gamma_{1} / \Gamma_{0} \ll 1$, and one timestep $\sigma$ is given by

$$
S_{r e g}=\Gamma_{1}[\sigma / 2] \Gamma_{0}[\sigma] \Gamma_{1}[\sigma / 2]=\Gamma[\sigma]+O\left(\epsilon \sigma^{3}\right)
$$

Like the Kepler problem, $\Gamma_{0}$ can be advanced analytically for special choices of $g(r)$.

Close encounters between bodies present another problem since $\epsilon$ is no longer small. Duncan et al. $(1998, A J 116,2067)$ overcome this by partitioning $H_{1}=V_{1}+V_{2}+\cdots$, where $V_{i}=0$ for $r>r_{i}$. Each $V_{i}$ has its own timestep $\tau_{i}$, such that larger $V_{i}$ have smaller $\tau_{i}$. One integration step consists of an infinitely nested set of substeps, each of which is evaluated only if $V_{i} \neq 0$. For example, when $V_{i}=0$ for $i>2$, one step is

$$
S_{C E}=V_{1}[\tau / 2]\left\{V_{2}[\tau / 2 M] H_{0}[\tau / M] V_{2}[\tau / 2 M]\right\}^{M} V_{1}[\tau / 2]=H[\tau]+O\left(\epsilon \tau^{3}\right)
$$

where $M$ is an integer.

Alternatively, Chambers $(1999, M N R A S$ 304, 793) partitions the perturbation terms between the two parts of the Hamiltonian, such that $H_{A}=H_{0}+H_{1}(1-P)$ and $H_{B}=H_{1} P$, with the partition function satisfying $P \rightarrow 1$ when $H_{1} \ll H_{0}$, and $H_{1} P \ll H_{0}$. One step including close encounters is

$$
S_{C E}=H_{B}[\tau / 2] H_{A}[\tau] H_{B}[\tau / 2]=H[\tau]+O\left(\epsilon \tau^{3}\right)
$$

where some terms in $H_{A}$ must be advanced numerically during an encounter.

To date, the general problem of how to combine close-encounter strategies with time regularization for eccentric orbits remains unsolved. 


\subsection{Orbit determination and impact risk}

\section{Author of the report: A. MILANI}

There is no such thing as the orbit of an asteroid determined by the observations: there is a confidence region in the phase space containing all the orbits compatible with the available astrometry. A rigorous definition of such a confidence region requires to adopt a statistical model of the observational errors. For the orbit of spacecraft it is customary to use the linearized Gaussian theory, assuming normal distribution of errors. Legitimate doubts on the applicability of the normal distribution hypothesis to the small number of astrometric observations of a single opposition asteroid have for a long time discouraged the use of a rigorously defined confidence region. That this situation is not satisfactory is now perceived, and recently some efforts have been done to introduce estimations of the orbital uncertainties based upon rigorous algorithms, with no subjective judgment. The limitations of the Gaussian algorithm due to the nonlinearity of the orbit determination problem have essentially been solved with Monte Carlo and semilinear methods (Muinonen and Bowell 1993, Icarus 104, 255; Muinonen et al. 1994, PSS 42, 307; Milani 1999, Icarus 137, 269; Chodas 1999, AAS/AIAA ASC, Girdwood, AK). Work to determine a reliable statistical model of the observation errors, based upon the a posteriori statistics of the past performance of each observatory, is now under way (Carpino 1997, http://schubert.brera.mi.astro.it/ carpino/orbfit/weights/; Williams, 1999, http://cfa-www.harvard.edu/iau/special/residuals.txt; Milani et al./ 1999, http://newton.dm. unipi.it) although not much has appeared in print so far.

The knowledge of the confidence region for the orbit of an asteroid/comet has two main applications: identification and close approach monitoring. The most efficient way to improve the orbit of a lost asteroid is by identification with another asteroid (observational resources required: zero). Literature on the identification problem is almost nonexistent (apart from Marsden 1985, in Asteroids, Meteors Comets II, Lagerkvist, C.-I. et al. eds., 3; Kristensen 1992, AA 262, 606; Sansaturio et al.1996, Dynamics, Ephemerides and Astrometry of the Solar System, Ferraz-Mello, Morando and Arlot Eds., Kluwer, 193). If the confidence region of two orbits intersect, then the two orbits could be identified; this orbit identification is a difficult problem because of the nonlinear effects which cannot be handled by computational brute force when working on catalogs of tens of thousands orbits, although some results have been obtained (Milani et al. 1999, Icarus, submitted). If the confidence region of an asteroid projected on the sky (at some time) contains an observation, then the observation can be attributed to the orbit. This attribution procedure has so far provided most of the identifications (mostly due to Marsden and Williams, Nakano, Doppler and Gnadig, Milani and Sansaturio); but there is no published paper explaining the algorithms being successfully used.

Close approaches of an asteroid/comet to the Earth can be predicted in a deterministic way as long the uncertainty in the position at close approach is much less than the closest approach distance. Even if the position uncertainty is much more than the distance, the asteroid cannot pass closer to the Earth than the Minimum Orbital Intersection Distance (MOID), the distance between the osculating ellipses (apart from gravitational focusing, which is small for typically fast encounters). Failure to appreciate this geometric constraint led to an unfortunate impact scare for the asteroid $1997 \mathrm{XF}_{11}$ (Chodas \& Yeomans 1999, 21st Annual AAS, Breckenridge, CO, Milani and Valsecchi 1999, Icarus 140; Muinonen 1999, The dynamics of the small bodies of the solar system: A major key to solar system studies, A. E. Roy and B. Steves, Eds., Kluwer). However, if the MOID is small, the close approach could be at a small distance and could result in a large perturbation in the asteroid semimajor axis. If the mean motion after the encounter is close to a small integer resonance $h / k$ with the Earth, then the relative positions approximately repeat after $k$ years and $h$ periods of the asteroid, and a new close approach could take place (Marsden 1999, JBAA $109,39)$. Such a resonant return could result in many close approaches, each of which could spawn its own cascade of resonant returns (Milani et al. 1999b) which can be completely monitored only by brute force, following numerically many alternate orbits (Chodas 1999, 
AAS/AIAA ASC, Girdwood, AK, Milani et al. 1999, Icarus, submitted). The region to be sampled with test orbits is the confidence region, and the nonlinearity problems arising from poorly observed asteroids compounds the difficulty of the close approach monitoring problem. Also the problem of the possible relevance of non gravitational perturbations in the prediction of possible impacts (for comets, but also possibly for some asteroids) remains to be fully addressed.

\subsection{Non-Integrable Galactic Dynamics}

\section{Author of the report : D. MERRITT}

Isolated galaxies have historically been viewed as integrable systems in which every orbit respects three isolating integrals of the motion, at least on time scales short compared to two-body relaxation times. This picture is a natural one in the case of spherical or axisymmetric galaxies, whose densities can always be reproduced using distribution functions that depend on just the classical integrals of motion. This was the point of view adopted by Jeans $(1915, M N R A S \mathbf{7 6}, 70)$, who expressed his "most general law of distribution" as $f=f\left(E, L_{z}\right)$, a function of the energy and angular momentum in an axisymmetric potential./ Motion in non-axisymmetric potentials conserves only the energy in general, and it was largely for this reason that triaxial geometries for elliptical galaxies were for so long ignored by astronomers - in spite of G. Kuzmin's demonstration, already in 1973 (in "The Dynamics of Galaxies and Star Clusters," T. B. Omarov ed., 71), that inhomogeneous triaxial mass models with integrable potentials exist. M. Schwarzschild's numerical construction of a self-consistent triaxial model in $(1979, A p J$ 232, 236) - and more importantly, his demonstration that most of the orbits were effectively regular - opened the door to a much wider class of galactic models, while at the same time leaving intact Jeans's view of galaxies as integrable systems.

The triaxial models of Kuzmin and Schwarzschild had large, constant-density cores in which the motion is essentially that of a simple harmonic oscillator. But observations in the last two decades have demonstrated that the density profiles of real elliptical galaxies are very different: the stellar density continues to rise, roughly as a power law, into the smallest observable radii (Crane et al. 1993, $A J$ 106, 1371 and Gebhardt et al. 1996, $A J 112,105$ ). There is also growing evidence that supermassive black holes are generic components of galactic nuclei (Kormendy and Richstone 1995, ARAA 33, 581). The orbital motion in a non-axisymmetric galaxy with a central density cusp or black hole can be very different from the motion in models like Kuzmin's and Schwarzschild's. Many orbits - particularly the box orbits that visit the center and which are crucial for maintaining triaxial shapes - are rendered chaotic (Gerhard and Binney 1985, MNRAS 216, 467), and even the orbits that remain regular are strongly influenced by resonances which play no role in the integrable models.

Non-integrability has thus come to be seen as a generic property of galactic potentials, and much of the research in galactic dynamics over the last few years has focussed on the consequences of non-integrability for the structure and evolution of stellar systems.

Phase-space structure of triaxial potentials In an integrable potential with $N$ degrees of freedom (DOF), all trajectories are regular and confined to $N$-dimensional tori. Motion around a torus occurs at a rate determined by a constant frequency vector $\left(\omega_{1}, \omega_{2}, \ldots, \omega_{N}\right)$. Realistic potentials with more than one DOF are rarely integrable and the motion is more complex. While the KAM theorem guarantees that most of the original tori will persist when an integrable potential is slightly perturbed, even under small perturbation a large part of the phase space will be influenced by resonances. A resonant torus is one that satisfies (one or more) conditions of form $\mathbf{n} . \omega=0$ between the $N$ fundamental frequencies. In the vicinity of a stable resonant torus, motion is still regular and the orbits have shapes determined by the order of the resonance - often very different from the shapes of orbits in the integrable potential./ In the vicinity of unstable resonant tori, trajectories are usually chaotic. 
The phase space of triaxial potentials is structured by the $3 \mathrm{D}$ resonant tori, which obey a condition on the three fundamental frequencies of the form $n_{1} \omega_{1}+n_{2} \omega_{2}+n_{3} \omega_{3}=0$. Such a relation does not imply that the orbit is closed, as in 2DOF, but rather that it is thin, densely filling a sheet in configuration space (Merritt and Valluri 1999, $A J$ 119). The KAM theorem predicts that in 3DOF systems as in 2DOF systems, the resonant tori are the regions around which the global structure of phase space may be strongly modified. In this sense, thin orbits play a similar role in three dimensions to the role played by periodic orbits in two dimensions.

A set of tools for recovering the $\omega_{i}$ was developed by J. Laskar ("NAFF," or Numerical Analysis of Fundamental Frequencies; $1988 A A 198,341$ ). A number of investigators applied Laskar's or similar algorithms to motion in triaxial models with realistic mass distributions (Papaphilippou and Laskar 1998, AA 329, 421; Carpintero and Aguilar 1998, $M N R A S$ 298, 1; Valluri and Merritt, $A p J$ 506, 686; Wachlin and Ferraz-Mello 1998, $M N$ $R A S \mathbf{2 9 8}, 22)$. The phase space of triaxial potentials is astonishingly complex, especially in regions occupied by box-like orbits, orbits with stationary points that pass near the center. Such orbits are found to be generically thin and associated with a $3 \mathrm{D}$ resonance; orbits that pass sufficiently close to the center are generally chaotic. Thus in realistic triaxial potentials, there are essentially no regular, volume-filling box orbits of the sort shown by Schwarzschild to be so important for maintaing triaxial shapes.

Stochasticity An early indication of the importance of stochasticity in triaxial potentials was the discovery that some of the orbits in Schwarzschild's triaxial model from 1979 yielded different occupation numbers when integrated using a different computer (Merritt 1980 , ApJS 43, 435), a consequence of the exponential divergence of nearby chaotic orbits. Schwarzschild's model had a large constant-density core, making it qualitatively similar to Kuzmin's exactly integrable model. In triaxial potentials with central singularities, the chaos can be much more pervasive. A number of techniques, developed mostly in other fields, have been used to evaluate the stochasticity of orbits in such potentials. Udry and Pfenniger $(1988, A A 198,135)$ computed all six Liapunov exponents for orbits in triaxial potentials based on Hubble's density law, and the same algorithm was applied to studies of motion in triaxial potentials with more realistic density profiles (Merritt and Fridman 1996, $A p J 460,136$ ) and central black holes (Merritt and Valluri 1996, ApJ 471, 82). Laskar's NAFF algorithm yields a natural measure of the stochasticity in the form of the change in a "fundamental frequency" between two successive integration intervals. This technique was applied in a number of studies to non-axisymmetric potentials (Papaphilippou and $\mathbf{J}$ Laskar 1996, $A A$ 307, 427; 1998, $A A$ 329, 421; Valluri and Merritt, $A p J$ 506, 686).

A basic result is that the influence of a central singularity can extend far beyond the nucleus of a non-axisymmetric galaxy. A central point mass divides the phase space of a triaxial potential into three regions depending on distance from the center. In the innermost region, the potential is essentially Keplerian and the motion is regular (Sridhar and Touma $1999, M N R A S 303,483$ ). This region extends to roughly the radius $r_{g}$ at which the enclosed stellar mass is a few times the black hole mass. Beyond this radius, the black hole acts as a scattering center, rendering almost all of the center-filling orbits stochastic. This "zone of chaos" extends outward from a few times $r_{g}$ to a radius where the enclosed stellar mass is $\sim 10^{2}$ times the black hole mass. At still larger radii, the phase space is a complex mixture of chaotic and regular trajectories, dominated by resonances, as discussed above. The transition between the intermediate and outer regions may be understood in terms of the destruction of the last remaining resonant tori by the black hole (Merritt and Valluri 1999, $A J$ 118).

Preliminary indications (Valluri, in "Galaxy Dynamics," 1999, ASP CS 182, 195) are that moderate figure rotation tends to modify this picture only by reducing the number of stable resonances.

Collisionless relaxation Stochasticity introduces a new type of relaxation into stellar dynamics that is qualitatively different from both "phase mixing" and "violent relaxation" (as the latter is usually defined). An initially localized ensemble of points in stochastic phase 
space will diverge exponentially; because the motion is essentially random, the probability of finding a point anywhere in the accessible phase-space region tends toward a constant, and so the ensemble density tends toward a uniform value (H. Kandrup and M. Mahon 1994, Phys. Rev. E., 49, 3735). This "chaotic mixing" differs from phase mixing in having a characteristic time scale, roughly the Liapunov time, and in being effectively irreversible - it represents a bona fide entropy increase. Chaotic mixing differs also from "violent relaxation" in the sense that it can occur even in fixed potentials; indeed one can usefully redefine "violent relaxation" as chaotic mixing in time-dependent potentials (H. Kandrup 1998, ApJ 500, 120).

The rate of chaotic mixing depends strongly on the structure of phase space. In globally chaotic regions - e.g. the "zone of chaos" in a triaxial potential - mixing is rapid, taking place in just a few crossing times. In phase-space regions containing mixtures of stochastic and regular trajectories, mixing time scales can be arbitrarily long (Kandrup $1998, M N R A S 301,960)$, though the mixing time is generally of order $\sim 10^{2}$ crossing times or less throughout most of a triaxial galaxy with a central point mass (Merritt and Valluri 1996, ApJ 471, 82).

Chaotic mixing converts all of the stochastic trajectories at a given energy into a single invariant ensemble. In weakly chaotic regions, such ensembles may have well-defined shapes, similar to that of regular orbits. Such components represent bona-fide density components for stationary or quasi-stationary galaxies, a realization that has led to a re-formulation of Jeans's theorem for non-integrable potentials (Kandrup 1998, MNRAS 299, 1139). In strongly chaotic regions, the invariant ensembles tend to be nearly spherical, mimicking the equipotential surface, and hence of little use in self-consistently reconstructing the density.

Self-consistency The case for triaxiality received a considerable boost from the demonstrations of Schwarzschild $(1979, A p J$ 232, 236) and Statler $(1987, A p J 321,113)$ that self-consistent triaxial equilibria could be constructed, at least in mass models with large cores. However subsequent observational studies found little evidence for significant triaxiality among real elliptical galaxies (e.g. Franx et al. 1991, ApJ 383, 112), prompting a re-analysis of the theoretical arguments. Schwarzschild (ApJ, 409, 563, 1993) repeated his pioneering self-consistency study using a scale-free, $\rho \sim r^{-2}$ density law and found that many of the box orbits were rendered stochastic by the central singularity, implying limits on the allowed axis ratios of a triaxial figure. Non-scale-free models with power-law central density cusps were likewise found to be consistent with only moderate departures from axisymmetry if the stochastic orbits were excluded (Merritt 1997, ApJ 486, 102).

These self-consistency studies were based on mass models lacking central black holes; furthermore the treatment of stochastic orbits in these studies is open to criticism. Omitting such orbits entirely is unjustified, since weakly chaotic trajectories can mimic regular orbits for long times; but it is not easy to determine which parts of stochastic phase space are likely to be uniformly populated after a given elapsed time without carrying out a full timedependent calculation. $N$-body studies are therefore an indispensable guide, and recently some special-purpose $N$-body algorithms have been applied to the problem. In the triaxial geometry, one finds (Merritt and Quinlan 1998, $A p J$ 498, 625) that the growth of a central point mass causes the surrounding galaxy to evolve to almost complete axisymmetry; the evolution time scale is of order a crossing time when the "black hole" mass exceeds $\sim 2.5 \%$ of the stellar mass, and shorter than a Hubble time for mass fractions of $\sim 0.3 \%$. The $N-$ body evolution rates observed in this study were similar to those inferred from the studies of mixing in fixed potentials cited above.

It is intriguing that black holes in real galaxies never exceed $\sim 2-3 \%$ the masses of their host spheroids (Kormendy et al. 1996, ApJ 459, L57; Cretton and van den Bosch $1999, A p J \mathbf{5 1 4}, 704)$, just the mass fraction that induces a rapid transition to axisymmetry in the $N$-body models. This coincidence suggests a link between the growth of black holes and the shapes of their host spheroids (Merritt and Quinlan 1998, ApJ 498, 625; Sellwood and Moore 1999, ApJ 510, 125). 
3. MAJOR SCIENTIFIC CONFERENCES 1997-1999

- IAU Colloquium 172 : Impact of modern dynamics in astronomy, Namur, Belgium, July 6-11, 1998

- Third International Symposium on Classical and Celestial Mechanics, Velikie Luki, Russia, August 23-28, 1998

- IAU Colloquium 173 : Evolution and source regions of asteroids and comets, Tatranska Lomnica, Slovak Republic, August 24-28, 1998

- European Astronomical Society, JENAM 97 : New trends in Astronomy and Astrophysics, Thessaloniki, Greece

- NATO : The Dynamics of Small Bodies in the Solar System: A Major Key to Solar System, Maratea, Italy, June 29 - July 12, 1997

- 4th Yugoslav-Romanian Astronomical Meeting, Belgrade, Yugoslavia, 1998.

\section{MAJOR PUBLICATIONS 1997 - 1999}

- The Dynamical Behaviour of our Planetary System R. Dvorak and J. Henrard, April 1997, Kluwer, 0792345487.

- Impact of Modern Dynamics in Astronomy J. Henrard and S. Ferraz-Mello, August 1999, Kluwer, 0792358422.

- New Foundations for Classical Mechanics (2nd Ed), Hestenes, October 1998, Kluwer, 0792355148.

- The Dynamics of Small Bodies in the Solar system: A Major Key to Solar Systems studies, Steves, December 1998, Kluwer, 0792354664.

- Dynamics and Astrometry of Natural and Artificial Celestial Bodies (IAU Coll. 165), I. M. Wytrzyszczak, J.H. Lieske and R. A. Feldman, July 1997, Kluwer, 0792345746.

- Dynamics of Comets and Asteroids and Their Role in Earth History, S. Yabushita and J. Henrard, October 1998, Kluwer, 0792352122.

- Celestial Dynamics at High Eccentricities, V. A. Brumberg and E. V. Brumberg, 1999, Gordon and Breach Science, 9056992120.

- Impacts on Earth, D. Benest and C. Froeschlé, 1998, Springer, 3540642099

- Generating Families in the Restricted Three Body Problem, M. Hénon, 1997, Springer, 3540638024 .

- New trends in Astronomy and Astrophysics : JENAM 97, J.D. Hadjidemetriou and J.H.Seiradakis, 1997, Hellenic Astronomical Society, 9604314408.

- Analysis and Modelling of Discrete Dynamical Systems, D. Benest and C. Froeschlé, 1998, Gordon and Breach Science, 9056996258.

Claude Froeschlé

President of the Commission 